\title{
Cardiac Tamponade in Adrenoleukodystrophy with Addison's Disease
}

\author{
Mafalda Sá Pereira, Raquel Freitas, Pedro Correia Azevedo \\ Hospital Garcia de Orta, Almada, Portugal
}

Received: 03/06/2021

Accepted: 09/06/2021

Published: 08/07/2021

How to cite this article: Sá Pereira M, Freitas R, Correia Azevedo P. Cardiac tamponade in adrenoleukodystrophy with Addison's disease. EJCRIM 2021;8: doi:10.12890/2021_002691.

Conflicts of Interests: The authors declare there are no competing interests.

This article is licensed under a Commons Attribution Non-Commercial 4.0 License

\section{ABSTRACT}

Cardiac tamponade is a life-threatening medical emergency and can arise in many clinical situations.

We present the case of a 59-year-old man with adrenoleukodystrophy and Addison's disease who was admitted to the emergency department with severe abdominal pain that turned out to be cardiac tamponade of unknown aetiology.

An association between cardiac tamponade and Addison's disease has been reported in the literature, so this aetiology should be considered in the differential diagnosis for patients presenting with unexplained cardiac tamponade.

\section{LEARNING POINTS}

- Cardiac tamponade can be associated with Addison's disease in rare cases.

- A high level of suspicion is essential, as it can mimic many medical conditions.

- Emergent pericardiocentesis is the required treatment.

\section{KEYWORDS}

Pericardial disease, adrenal disorders, emergency medicine

\section{BACKGROUND}

Cardiac tamponade is caused by the accumulation of fluid in the pericardial space resulting in reduced ventricular filling and subsequent haemodynamic instability. It is a medical emergency requiring emergent pericardiocentesis. Symptoms and signs lack both sensitivity and specificity. A high index of suspicion with a proper diagnostic protocol can lessen the concomitant morbidity and mortality.

Adrenoleukodystrophy is linked to the X chromosome (X-ALD) and is the most common peroxisomal disorder. It has a variable phenotypic presentation, affecting the nervous system, adrenal cortex and testicular function. X-ALD is caused by mutations in the ABCD1 gene located at Xq28. The incidence of X-ALD has been reported to be 1:21,000 in males and 1:14,000 in females ${ }^{[1]}$. Primary adrenal insufficiency (Addison's disease) is reported to develop in up to $86 \%$ of males with X-ALD. Addison's disease (AD) is caused by a deficiency of glucocorticosteroids and mineral corticosteroids.

\section{CASE DESCRIPTION}

A 59-year-old man was admitted to the emergency department with severe abdominal pain. On admission, he complained of severe epigastric pain with radiation to the back that had started 12 hours previously, with no other accompanying symptoms.

The patient had familial adrenomyeloneuropathy with spastic paraparesis and AD, diagnosed years previously. He did not have cardiovascular risk factors, except for dyslipidaemia. 
On physical examination, he was haemodynamically unstable, with blood pressure of $89 / 46 \mathrm{mmHg}$, heart rate of 110 bpm, respiratory rate of $19 \mathrm{rpm}$ and body temperature of $37.0^{\circ} \mathrm{C}$. He was distressed and pale, with mottled skin, muffled heart sounds, elevated neck veins, pain on abdominal examination and diffuse abdominal distension.

Arterial blood gas showed hypoxaemia $\left(\mathrm{pO}_{2} 65.5 \mathrm{mmHg}\right)$ and hyperlactaemia $(2.1 \mathrm{mmol} / \mathrm{l})$.

An electrocardiogram (ECG) showed low voltage QRS in the precordial leads. Abdominal ultrasound did not show any signs of pathology. A computerized tomography (CT) scan revealed a pericardial high-density effusion with reduced diastolic filling of the right atrium and right ventricle and bilateral pleural effusion (Fig. 1).

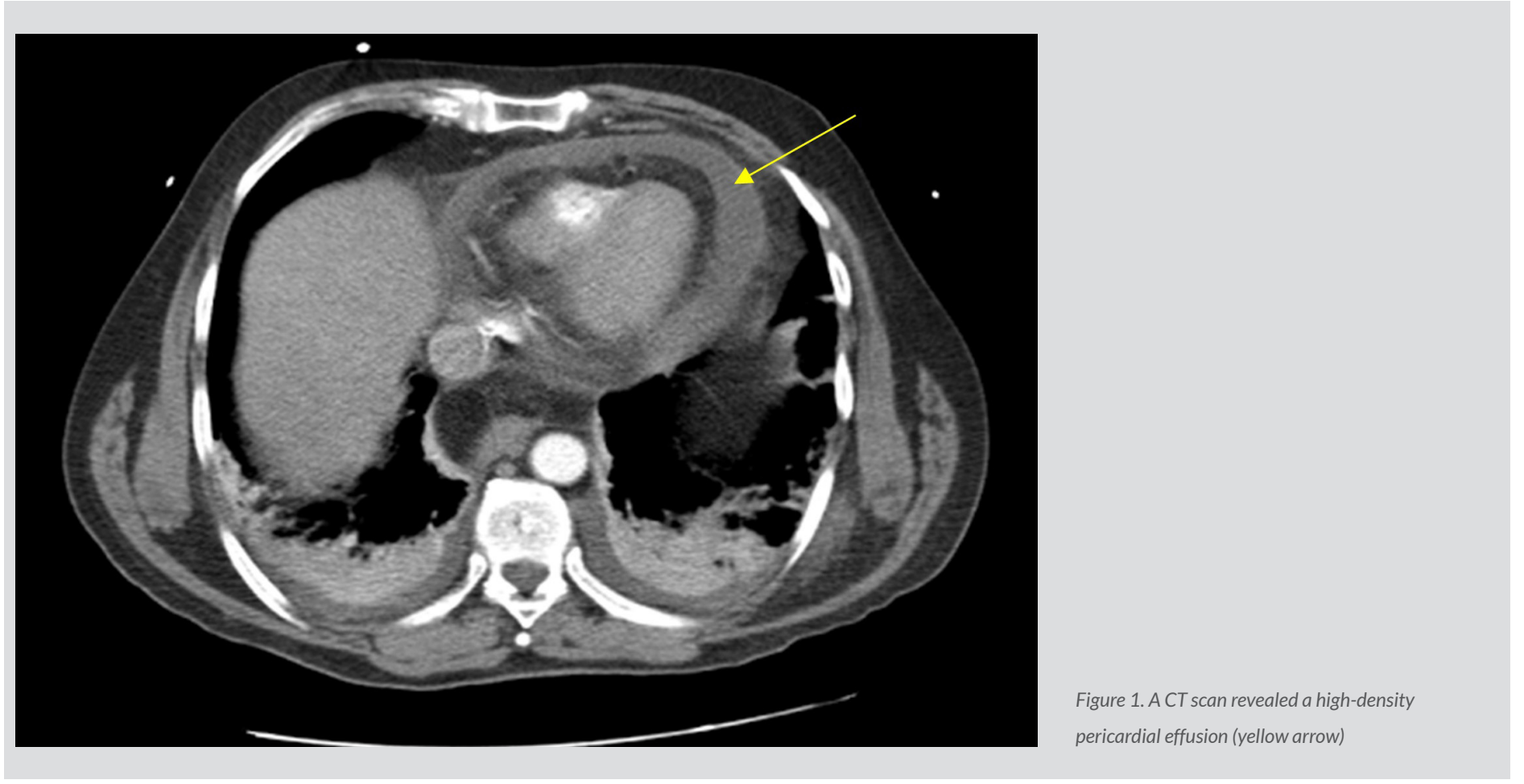

Bedside echocardiography revealed pericardial thickening and a moderate to severe pericardial effusion with indirect signs of cardiac tamponade and low transmitral and transtricuspid gradients. Images were not obtained.

Emergent pericardiocentesis was performed and approximately $500 \mathrm{ml}$ of yellow pericardial fluid was evacuated. A large drain was placed in the pericardial cavity with a further $200 \mathrm{ml}$ of fluid evacuated.

Laboratory investigations showed leucocytosis (27.0×10\%/l), with 73.6\% neutrophils, elevated C-reactive protein (CRP) (15.76 mg/dl), acute renal dysfunction (creatinine $2.4 \mathrm{mg} / \mathrm{dl}$ ) and a cardiac marker troponin T level of $30 \mathrm{ng} / \mathrm{l}$.

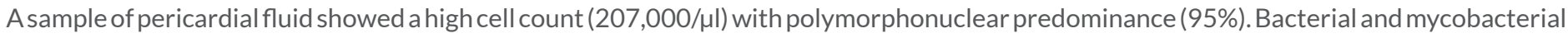
cultures of blood and pericardial fluid were all negative. Autoimmune investigations were negative (ANA, anti-dsDNA, ENA) as was serology for HIV.

The patient was admitted to the intensive care unit where he remained for 5 days. He required noradrenaline perfusion to achieve haemodynamic stability. He started acetylsalicylic acid $1000 \mathrm{mg}$ per day, colchicine $1 \mathrm{mg}$ twice a day and empiric antibiotic therapy with ceftriaxone and vancomycin for 7 days.

Substantial improvement in symptoms and haemodynamic parameters was achieved and the patient was discharged after 14 days. Echocardiography re-evaluation showed a thin layer of pericardial effusion.

\section{DISCUSSION}

This is a very rare case of the coexistence of $A D$ and adrenoleukodystrophy, leading to cardiac tamponade.

Cardiac tamponade can be caused by any disease that involves the pericardium, including infection, autoimmune disease, neoplasm and radiation therapy. Acute pericardial effusion is mainly idiopathic in developed countries, and presumed to be of viral origin although specific causes have not been identified. 
Although in this case we did not identify a definite aetiology for the cardiac tamponade, an association between cardiac tamponade and concurrent $A D$ has been reported in the literature ${ }^{[2-4]}$. The underlying mechanism for pericardial effusion is possibly autoimmune inflammation of the pericardium, which leads to an acute inflammatory reaction and fluid accumulation ${ }^{[5]}$. Pericardial fluid accumulation can result in increased pericardial pressure, compression of the cardiac chambers and cardiac tamponade.

This case also highlights the importance of maintaining a high level of suspicion for cardiac tamponade, as it can mimic many other medical conditions. We emphasize the importance of physical examination and highlight the unusual presentation, as in this case the patient presented with acute and severe abdominal pain.

Cardiac tamponade can be a consequence of $A D$ and this condition should be considered in the differential diagnosis for unexplained cardiac tamponade.

Symptoms and signs of cardiac tamponade lack both sensitivity and specificity. Transthoracic echocardiography is the most important tool for diagnosis, drainage and follow-up. Cardiac tamponade is a medical emergency that requires emergent pericardiocentesis. A high index of suspicion with proper diagnostic investigations lessens the concomitant morbidity and mortality.

\section{REFERENCES}

1. Bezman L, Moser AB, Raymond GV, Rinaldo P, Watkins PA, Smith KD, et al. Adrenoleukodystrophy: incidence, new mutation rate, and results of extended family screening Ann Neurol 2001;49:512-517.

2. Alkaabi J, Chik C, Lewanczuk R. Pericarditis with cardiac tamponade and addisonian crisis as the presenting features of autoimmune polyglandular syndrome type II: a case series. Endocr Pract 2008;14:474-478.

3. Vryonidou A, Paschou SA, Dimitropoulou F, Anagnostis P, Tzavara V, Katsivas A. Cardiac tamponade in a patient with autoimmune polyglandular syndrome type 2. Endocrinol Diabetes Metab Case Rep 2017;2017:17-0097.

4. Manthri S, Bandaru S, Ibrahim A, Mamillapalli CK. Acute pericarditis as a presentation of adrenal insufficiency. Cureus 2018;10(4):e2474. doi: 10.7759/cureus.2474

5. Al-Mutairy M, Malaviya AN. Systemic lupus erythematosus with polyglandular autoimmune syndrome type II: report of an unusual case. Lupus 2001;10:445-447. 